\section{La importancia de las ciencias básicas en la formación del cirujano dentista}

\author{
The importance of basic science \\ in the training of dental surgeons
}

Angela Quispe-Salcedo 1,2,a

\author{
${ }^{1}$ Universidad Nacional Mayor de San Marcos. Facultad de Odonto- \\ logía. Lima, Perú. \\ 2 Universidad Científica del Sur. Facultad de Ciencias de la Salud, \\ Carrera de Estomatología. Lima, Perú. \\ a. Doctora en Ciencias Odontológicas. \\ Correspondencia: \\ Angela Quispe-Salcedo \\ Correo electrónico: angela.quispe@unmsm.edu.pe \\ Universidad Nacional Mayor de San Marcos, Facultad de Odonto- \\ logía. Calle Germán Amézaga 375. Lima 1, Perú. \\ Fecha de recepción: 20/08/18 \\ Fecha de aceptación: 28/08/18
}

Explicar la ciencia no es tarea sencilla. Desde una aproximación actual, la ciencia puede definirse como una descripción sistemática de un determinado fenómeno presente en la naturaleza ${ }^{1}$; donde la observación y la interpretación se interrelacionan para dar lugar a un nuevo conocimiento que se estandariza mediante el uso del método científico. Dentro de este contexto, la ciencia puede ser dividida en básica y aplicada, teniendo cada una características y objetivos distintos.

Las ciencias básicas tienen por objetivo principal entender cómo es que suceden y funcionan las cosas a nuestro alrededor, aumentando el conocimiento del ser humano respecto a su entorno ${ }^{2}$. Por ejemplo, el investigador que intenta averiguar la ultraestructura de la fibra de colágeno tipo I, abundante en la dentina, está haciendo investigación básica. Al contrario, la ciencia aplicada es aquella que contribuye a la creación de tecnologías o nuevos procesos, basados en el conocimiento generado por la investigación básica. Un ejemplo de investigación aplicada es el desarrollo de nanotecnologías para la liberación controlada de fármacos en el interior del cuerpo humano. Así, la interrelación de las ciencias básicas y aplicadas es fundamental para el progreso de la sociedad.

La formación del cirujano dentista durante el pregrado pone énfasis en el aprendizaje de conocimientos de la carrera que le permitan entender, reconocer y diagnosti- car las patologías que aquejan al sistema estomatognático. Asimismo, se enfoca en la adquisición y el desarrollo de habilidades y destrezas que permitan realizar exitosamente la diversidad de tratamientos para la resolución de las enfermedades bucodentales. No obstante, el conocimiento y aprendizaje de las ciencias básicas constituye también una parte importante en la malla curricular de todo programa de odontología; ya que es indiscutible la necesidad de interrelacionar estos conceptos con la parte clínica de la profesión.

En odontología, las ciencias básicas cumplen un rol fundamental para poder entender la complejidad del proceso salud-enfermedad que toma lugar en la cavidad bucal. El estudio de las ciencias básicas generales como la química, física o biología durante la etapa preuniversitaria, preparan al estudiante para adentrarse en los conocimientos básicos más relacionados a la odontología. De esta forma, los cursos que se enseñan en los primeros semestres de formación académica tales como anatomía humana y estomatológica, bioquímica general y aplicada, histología y embriología general y estomatológica, microbiología bucal, entre otros, sientan las bases necesarias para formar un profesional competente con la capacidad de integrar los conocimientos básicos y clínicos.

En un artículo publicado en el 2003, Baum reafirma la importancia de la incorporación de las ciencias biomé- 
dicas en la malla curricular de los programas de odontología en los Estados Unidos, a manera de respuesta frente a la tendencia actual de disminuir los contenidos de los cursos básicos, o eliminar algunos de ellos. El autor señala que el entendimiento de la ciencia y el desarrollo del pensamiento crítico, sin llegar a menospreciar las habilidades y técnicas de la odontología convencional, son necesarios para poder comprender la biología de los tejidos y la progresión de las enfermedades bucodentales; utilizando un pensamiento lógico y riguroso que favorece la atención clínica a los pacientes ${ }^{3}$.

Maldonado-Frías ${ }^{4}$ también hace un análisis de la importancia de los cursos de ciencias básicas en odontología, con énfasis en la enseñanza de la bioquímica. El texto concluye resaltando que la comprensión de la utilidad y aplicación de las áreas básicas en la educación odontológica contribuye a que los alumnos que cursan la carrera de cirujano dentista puedan tener una sólida preparación que les permita estar a la par con los cambios científicos y tecnológicos que se dan en la actualidad.

Los continuos avances en todas las áreas de la ciencia no son excluyentes a la odontología. Cada día se publican hallazgos científicos dilucidando conceptos fundamentales antes desconocidos para nuestra carrera en áreas relativamente "nuevas" como genética dental, microbiología molecular, biología del desarrollo craneofacial, proteómica aplicada, solo por mencionar algunas. Estos descubrimientos servirán de base para la creación de tecnologías que contribuyan al cambio hacia una odontología regenerativa basada en evidencias científicas. Conviene destacar el rápido avance en el estudio y caracterización de las células madre de origen dental, que, junto al desarrollo de materiales cada vez más biocompatibles, ha abierto paso a la ingeniería de tejidos para la restauración de estructuras dentales perdidas. Lamentablemente, a pesar del progreso de la ciencia y tecnología en nuestra profesión, la asimilación de esta información en el currículo de las escuelas de odontología, tanto en el Perú como en el extranjero, es aún bastante lenta ${ }^{5}$.

El Departamento de Ciencias Básicas de la Facultad de Odontología de la Universidad Nacional Mayor de San Marcos, ha sido el pionero en la enseñanza de las ciencias básicas odontológicas en el Perú. La plana docente tiene amplia experiencia en sus respectivas materias, que garantizan al alumno una sólida formación en el área básica durante los primeros ańos de vida universitaria. Sin embargo, es necesario que más cirujanos dentistas se capaciten en las áreas básicas para continuar brindando educación de calidad a los futuros profesionales odontólogos de nuestro país. Esto último se ve afectado por la ausencia en nuestro medio de programas de posgrado, ya sean maestrías o doctorados en ciencias básicas odontológicas, constituyendo una gran desventaja que ha perjudicado la formación de nuevos docentes del área básica.

En suma, queda claro que las ciencias básicas son el pilar en la educación del cirujano dentista. La revalorización de los cursos básicos dictados en los primeros semestres es tarea de todos y se debe trabajar con el objetivo de despertar en el alumno la curiosidad por el conocimiento de los fundamentos biológicos que guían los tratamientos clínicos. Por otro lado, el docente debe estar al tanto de los adelantos en sus respectivas materias y recibir capacitación continua que le permita transmitir el entusiasmo por las ciencias a sus estudiantes, incentivando la lectura de artículos científicos que puedan resolver sus inquietudes y al mismo tiempo desarrollar su pensamiento crítico. Es a través de la ciencia que un país puede desarrollarse, y en nuestro caso, la enseñanza de las ciencias básicas odontológicas garantiza a las futuras generaciones el ejercicio de una odontología basada en fundamentos científicos, contribuyendo al progreso de nuestra profesión.

\section{Referencias bibliográficas}

1. Richards WT. A definition of science. J Chem Educ.1928;5(7):874

2. Pielke R. In Retrospect: Science - The Endless Frontier. Nature. 2010;466(19):222-23.

3. Baum BJ. Can biomedical science be made relevant in dental education? A North American perspective. Eur J Dent Educ. 2003;7:49-55.

4. Maldonado-Frías S. Bioquímica. La importancia de las áreas básicas en la odontología. Rev Odont Mex. 2013;17(2):74-75.

5. Iacopino AM. The influence of "new science" on dental education: current concepts, trends, and models for the future. J Dent Educ. 2007;71(4):450-62. 